Erratum

\title{
Erratum to "Montreal Cognitive Assessment Is Superior to Standardized Mini-Mental Status Exam in Detecting Mild Cognitive Impairment in the Middle-Aged and Elderly Patients with Type 2 Diabetes Mellitus"
}

\author{
Kannayiram Alagiakrishnan, ${ }^{1}$ Nancy Zhao, ${ }^{1}$ Laurie Mereu, ${ }^{1}$ \\ Peter A. Senior, ${ }^{1}$ and Ambikaipakan Senthilselvan ${ }^{2}$ \\ ${ }^{1}$ Department of Medicine, University of Alberta Hospital, B139 Clinical Sciences Building, Edmonton, AB, Canada T6G $2 G 3$ \\ ${ }^{2}$ School of Public Health, University of Alberta, Edmonton, AB, Canada T6G 2G3
}

Correspondence should be addressed to Kannayiram Alagiakrishnan; kalagiakri@aol.com

Received 16 March 2014; Accepted 1 April 2014; Published 28 April 2014

Copyright (C) 2014 Kannayiram Alagiakrishnan et al. This is an open access article distributed under the Creative Commons Attribution License, which permits unrestricted use, distribution, and reproduction in any medium, provided the original work is properly cited.

There are some errors on page 2 with the cut-off score for MCI based on MoCA score.

Under Methodology, in the last paragraph-5th line, there is an error, "A MoCA score of 26 or less is considered as MCI" which should read as "A MoCA score of less than 26 (25 or less) is considered as MCI." Under Statistical Analysisline 9 , there is another error, scores " $\leq 26$ " which should be " $<26$," and, in Tables 3 and 4 , the error is "total score $(\leq 26)$ " which should be " $(<26)$." 
TABLE 3: Abnormal scores on different domains of MoCA in both middle-aged (50-64 years) and elderly ( $\geq 65$ years) patients with and without MCI by the European consortium criteria.

\begin{tabular}{lccc}
\hline \multirow{2}{*}{ Abnormal score on domains } & \multicolumn{2}{c}{ European consortium criteria } & \\
& MCI present $(n=15)$ & MCI absent $(n=15)$ & $1(6.7 \%)$ \\
\hline Total score $(<26)$ & $7(46.7 \%)$ & $6(40 \%)$ & 0.04 \\
Visuo spatial & $8(53.3 \%)$ & $4(26.7 \%)$ & 0.46 \\
Language & $8(53.3 \%)$ & $2(13.3 \%)$ & 0.14 \\
Attention and calculation & $6(40 \%)$ & $3(20 \%)$ & $0.22^{*}$ \\
Abstraction & $6(40 \%)$ & $10(66.1 \%)$ & $0.43^{*}$ \\
Memory & $13(86.7 \%)$ & $0(0 \%)$ & $0.39^{*}$ \\
Orientation & $0(0 \%)$ & $2(13.3 \%)$ & - \\
Clock drawing & $6(40 \%)$ & 0.22 \\
\hline
\end{tabular}

With discrete variable, the differences in the proportions were tested by chi-square tests except that ${ }^{*}$ Fisher's exact test was used to test the differences in the attention and calculation, abstraction, and memory.

TABLE 4: Abnormal scores on different domains of MoCA in the middle-aged (50-64 years) patients with and without MCI by the European consortium criteria.

\begin{tabular}{lccc}
\hline Abnormal score on MoCA & \multicolumn{2}{c}{ European consortium criteria } & $P$ value \\
& MCI present $(n=11)$ & MCI absent $(n=11)$ & $1(9.1 \%)$ \\
Total score $(<26)$ & $4(36.4 \%)$ & $3(27.3 \%)$ & 0.31 \\
Visuo spatial & $4(36.4 \%)$ & $6(54.6 \%)$ & 1.00 \\
Language & $5(45.5 \%)$ & $1(9.1 \%)$ & 1.00 \\
Attention and calculation & $4(36.4 \%)$ & $3(27.3 \%)$ & 0.31 \\
Abstraction & $6(54.6 \%)$ & $7(63.6 \%)$ & 0.39 \\
Memory & $10(90.9 \%)$ & $0(0.0 \%)$ & 0.31 \\
Orientation & $0(0.0 \%)$ & $2(18.2 \%)$ & - \\
Clock drawing & $3(27.2 \%)$ & & 1.0 \\
\hline
\end{tabular}

${ }^{*}$ The differences in the proportions were tested by Fisher's exact test. 


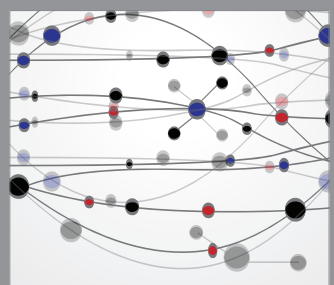

The Scientific World Journal
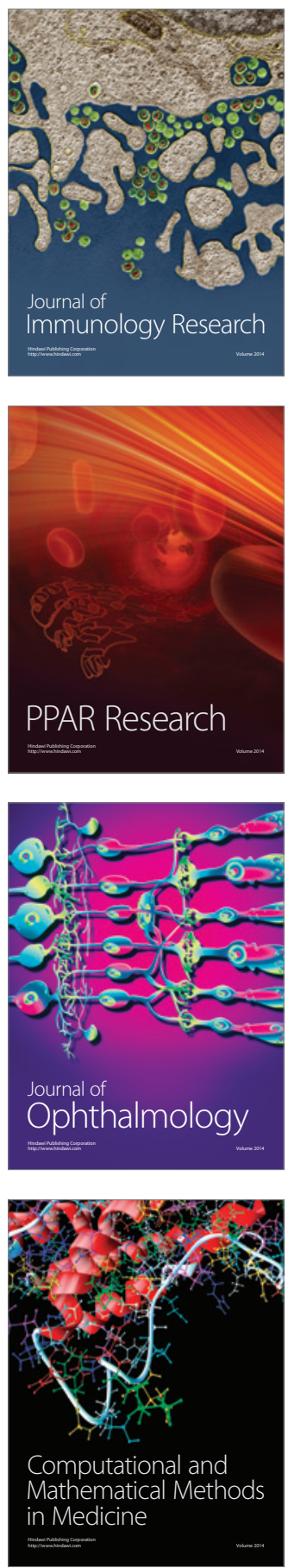

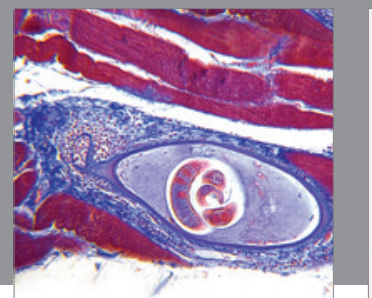

Gastroenterology

Research and Practice
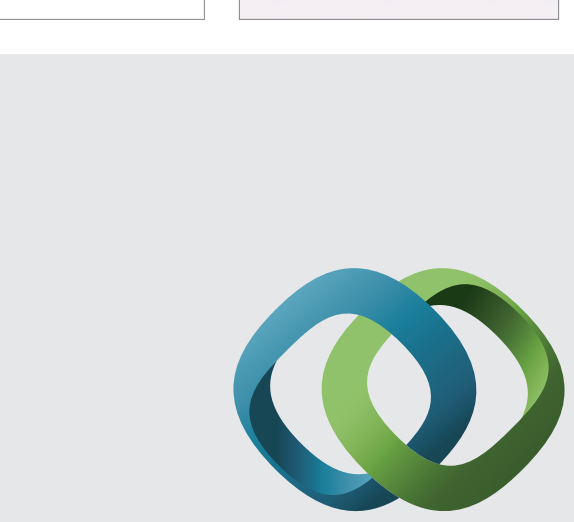

\section{Hindawi}

Submit your manuscripts at

http://www.hindawi.com
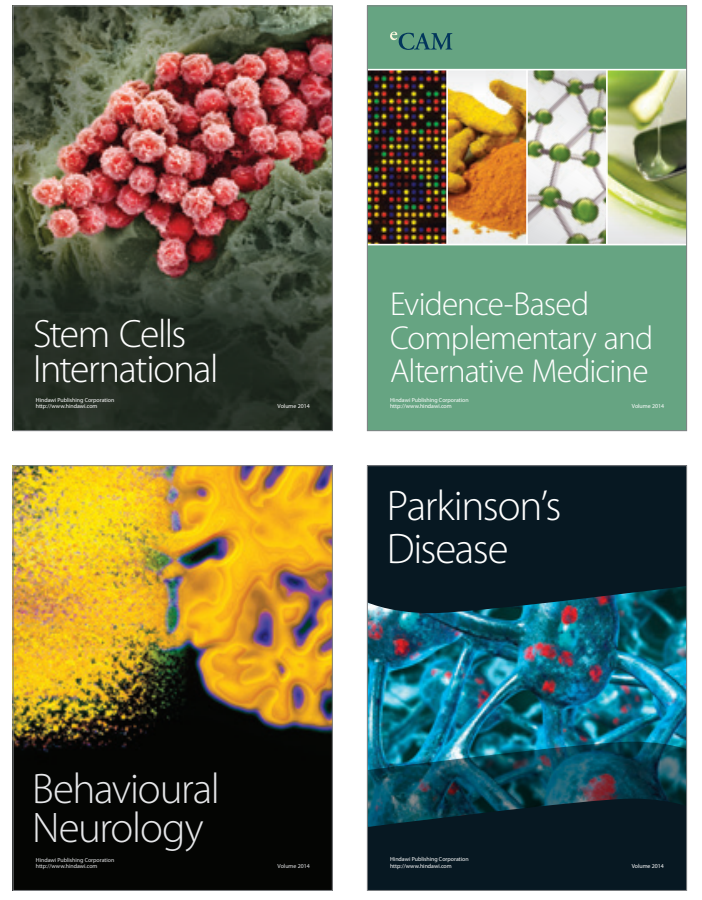
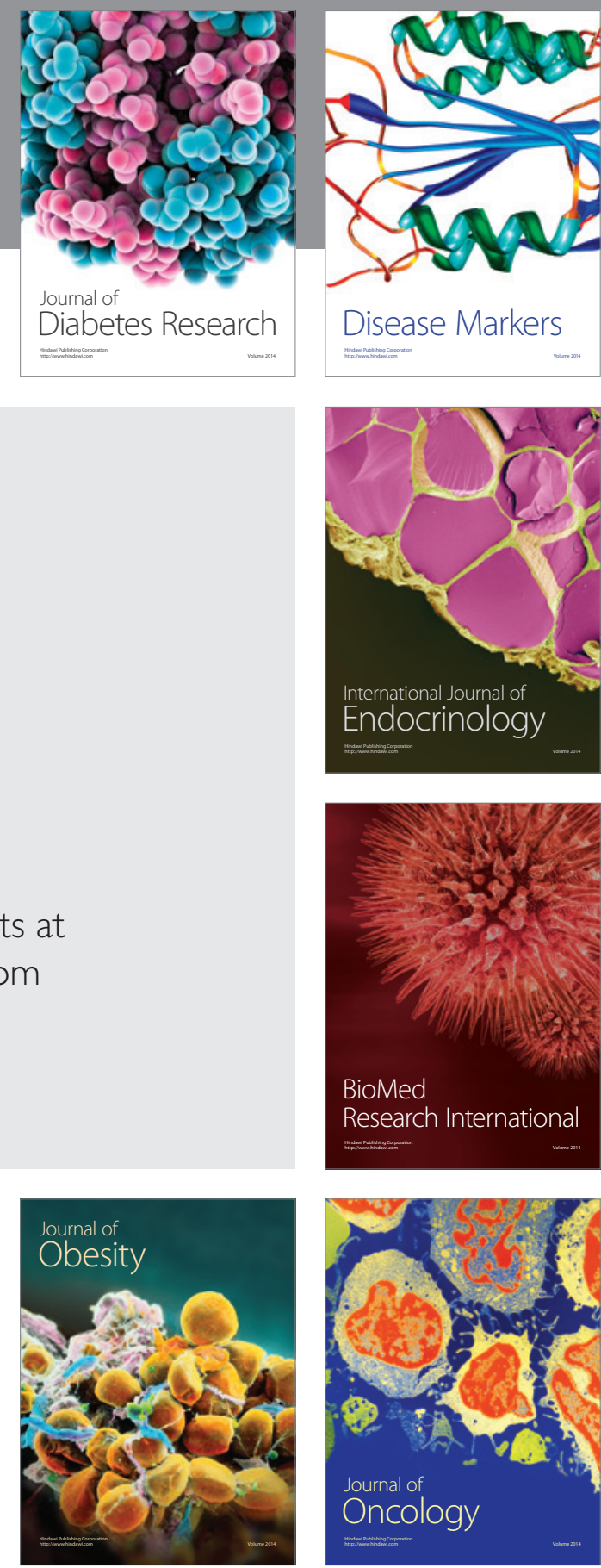

Disease Markers
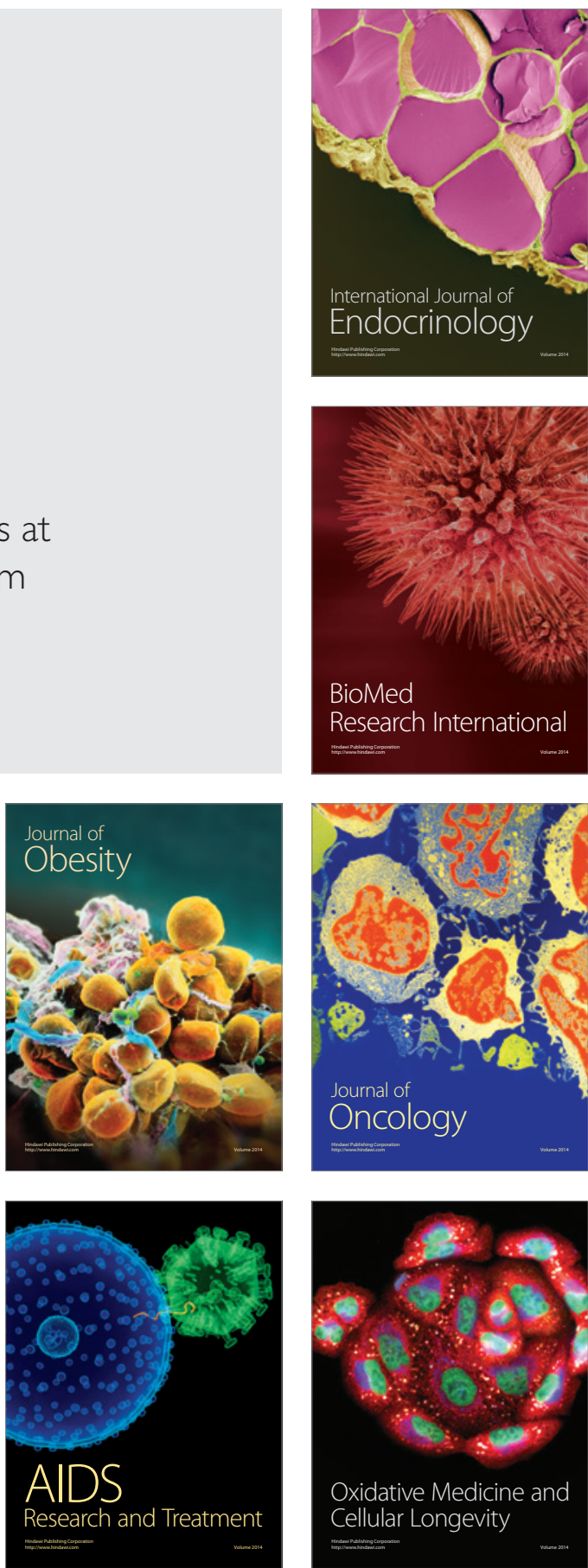